\title{
Quantum Measurements, Information and Entropy Production
}

\author{
Y.N. Srivastava \\ Dipartmento di Fisica \& INFN, Universita' di Perugia, I-06100 Perugia, Italy \\ G. Vitiello \\ Dipartmento di Fisica \& INFM, Universita' di Salerno, I-84100 Salerno, Italy \\ A. Widom \\ Physics Department, Northeastern University, Boston MA 02115 U.S.A.
}

\begin{abstract}
In order to understand the Landau-Lifshitz conjecture on the relationship between quantum measurements and the thermodynamic second law, we discuss the notion of "diabatic" and "adiabatic" forces exerted by the quantum object on the classical measurement apparatus. The notion of heat and work in measurements is made manifest in this approach, and the relationship between information entropy and thermodynamic entropy is explored.
\end{abstract}

03.65.Bz, 03.67.-a

\section{INTRODUCTION}

Thermodynamics has withstood the onslaught of the two revolutions in physical theory that have occurred during this century; i.e. relativity and quantum mechanics. Yet the microscopic basis of of thermodynamic laws remains unclear. What appears to be time reversal symmetry on the microscopic scale turns into time reversal asymmetry on the macroscopic scale. Our entire intuition concerning what constitutes the "forward direction in time" is based upon our observed experience of the second law "increase of entropy". Yet the foundations of this thermodynamic break with reversible time are at best poorly understood.

Notions of "quantum measurements" are also not very well understood. The working physicist usually does not wish to think too hard about such a basic problem. For guidance one consults (and repeats) the rules of Bohr. The rules are as follows: (i) A measurement is an interaction between a classical apparatus and a quantum object. (ii) All data are classical and thereby reside in the motion of a classical apparatus; and (iii) One may never look directly at a quantum object. If one asks experimentally what the quantum object is really doing, then the quantum object behaves in a classical fashion as an apparatus. If one asks theoretically what the quantum object is doing, then one risks being called a philosopher (or perhaps even worse). Bohr [1] forbid Einstein to ask what the quantum object was really doing, and no agreed upon answer has yet been formulated. Designing a great experiment using the Bohr rules is similar to creating a great sculpture employing a Lagrangian. The real art is in making those judgments that are not so well described by dry logical formal rules.

Landau and Lifshitz [2] [3], in their classical treatises on theoretical physics, attempted to connect the above questions concerning the nature of the second law of thermodynamics and the quantum picture of measurements; They conjectured that second law entropy increases are required for quantum measurements, and (conversely) that quantum measurements are responsible for the second law increase in entropy. A consequence of this conjecture is that the second law has no foundation in classical statistical thermodynamics. Quantum mechanics must be essential. However, Landau and Lifshitz (by their own evaluation) never really proved their conjecture. Alas, nor shall we. However, our purpose is to describe (in formal detail) the problems as they exist.

In Sec.2, the classical apparatus Lagrangian is defined. In Sec.3, we describe the interaction of this classical apparatus with the quantum object. We also introduce the notion of "diabatic" and "adiabatic" forces exerted by the quantum object on the classical apparatus. In Sec.4 the notion of quantum measurements via the apparatus Lagrangian renormalization is discussed. In Sec.5, the formalism is illustrated for the Stern-Gerlach experiment [4]. In this experiment, the data was sent [5] (in picture post card form) to Bohr with a message of congratulations for understanding quantum mechanics. Actually, what goes on in this experiment is (to this day) somewhat mysterious.

In Sec. 6, the notion of "work" and "heat" contributions for the energy changes in a measurement ensemble of quantum objects is explored in terms of adiabatic forces and diabatic (non-adiabatic) transition forces. For macroscopic bodies in the microcanonical ensemble, the quantum measurement decomposition of forces into those that perform work and those that change energies via heat production are shown to be equivalent to the thermodynamic decomposition of energy changes into heat and work. The proof, employing the microcanonical assumption, is provided in Sec.7. However, we make no claim to have derived the thermodynamic laws and their time reversal symmetry breaking quality. The situation is what it appeared to be about a century ago. The statistical ensembles (microcanonical or canonical) of Gibbs are eminently reasonable descriptions of macroscopic sys- 
tems. But no rigorous derivation from dynamics yet exists.

In Sec. 8 we discuss linear friction on the measurement apparatus induced by the quantum object. That no such friction term exists if one employs the projection postulate is noted in Sec.9. In Sec.10, the information entropy is introduced within the context of projective measurements. The relationship between information entropy and thermodynamic entropy is explored. In the concluding Sec.11, we assert that for quantum objects with an infinite number of degrees of freedom it is reasonable to invoke a non-unitary time development during the measurement process.

\section{THE CLASSICAL APPARATUS}

From a classical viewpoint, we describe the classical apparatus motion as a path on a manifold with local coordinates

$$
x=\left(x^{1}, \ldots, x^{n}\right) .
$$

The path $x(t)$ of the apparatus motion, were the apparatus not to interact with the quantum object, is determined by the Lagrangian

$$
L_{A}(x, v)=\frac{1}{2} \mu_{j k}(x) v^{j} v^{k}-V(x),
$$

where the velocity tangent vector $v=\left(v^{1}, \ldots, v^{n}\right)$ is defined by

$$
v^{k}=\left(d x^{k} / d t\right)
$$

and we employ the convention of implicit summation over twice repeated (covariant and contravariant) indices. If the apparatus does not interact with a quantum object, then the equations of motion read

$$
\frac{d}{d t}\left(\frac{\partial L_{A}}{\partial v^{k}}\right)=\left(\frac{\partial L_{A}}{\partial x^{k}}\right) .
$$

Quantum measurements are performed when the quantum object causes the apparatus to deviate from the above unperturbed classical paths. Such deviations may be described by a renormalization of the apparatus Lagrangian induced by an interaction with a quantum object.

\section{QUANTUM OBJECT}

The Hamiltonian of the quantum object will be viewed as an operator whose matrix elements depend on the apparatus coordinates,

$$
H(x)=\left(\begin{array}{cccc}
H_{11}(x) & H_{12}(x) & \ldots & H_{1 m}(x) \\
H_{21}(x) & H_{22}(x) & \ldots & H_{2 m}(x) \\
\ldots & \ldots & \ldots & \ldots \\
H_{m 1}(x) & H_{m 2}(x) & \ldots & H_{m m}(x)
\end{array}\right) .
$$

If the apparatus path $x(t)$ is given, then the quantum object is thought to be described by the Schrödinger equation

$$
i \hbar \frac{\partial}{\partial t}|\psi(t)\rangle=H(x(t))|\psi(t)\rangle .
$$

There is no conceptual problem about how a classical apparatus will change the state of a quantum object. The manner in which a quantum object changes the path of $x(t)$ is a bit more subtle.

The quantum object interacts with the classical apparatus in virtue of the variation of $H(x)$ with $x$; i.e. the force exerted on the apparatus by the quantum object is given by

$$
\mathcal{F}_{k}(x)=-\left(\frac{\partial H(x)}{\partial x^{k}}\right) .
$$

The force is evidently a quantum operator on the quantum system which is supposed to have an effect on the classical path $x(t)$. How this occurs is not at once obvious.

It is here convenient to invoke a unitary operator

$$
U^{\dagger}(x)=U^{-1}(x)
$$

which obeys

$$
W(x)=U^{\dagger}(x) H(x) U(x)
$$

with $W(x)$ diagonal,

$$
W(x)=\left(\begin{array}{cccc}
W_{1}(x) & 0 & \ldots & 0 \\
0 & W_{2}(x) & \ldots & 0 \\
\ldots & \ldots & \ldots & \ldots \\
0 & 0 & \ldots & W_{m}(x)
\end{array}\right) .
$$

From Eqs.(6), (9) and

$$
|\psi(t)\rangle=U(x(t))|\Psi(t)\rangle
$$

it follows that

$$
i \hbar \frac{\partial}{\partial t}|\Psi(t)\rangle=\mathcal{H}(x(t), v(t))|\Psi(t)\rangle
$$

where

$$
\mathcal{H}(x, v)=W(x)-v^{k} P_{k}(x)
$$

and

$$
P_{k}(x)=i \hbar U^{\dagger}(x) \frac{\partial U(x)}{\partial x^{k}}=-i \hbar \frac{\partial U^{\dagger}(x)}{\partial x^{k}} U(x) .
$$

From Eqs.(12) and (13), it follows that $\mathcal{H}(x, v)$ is the quantum object Hamiltonian when the interaction is with an apparatus whose motion exhibits coordinates $x$ and velocities $v$. The force operator acting on the apparatus follows from Eqs.(9) and (14) to be 


$$
U^{\dagger}(x) \mathcal{F}_{k}(x) U(x)=F_{k}(x)+f_{k}(x)
$$

where

$$
F_{k}(x)=-\left(\frac{\partial W(x)}{\partial x^{k}}\right)
$$

is the "adiabatic" part of the force, and

$$
f_{k}(x)=-\frac{i}{\hbar}\left[W(x), P_{k}(x)\right]
$$

is the "diabatic", i.e. "non-adiabatic", part of the force.

\section{ADIABATIC MEASUREMENT LAGRANGIANS}

The object Hamiltonian in Eq.(5) allows for the notion of a quantum measurement as a renormalization of the apparatus Lagrangian in Eq.(2). Explicitly, if one defines a measurement basis for the quantum object employing the adiabatic energy levels of Eqs.(9) and (10),

$$
H(x)\left|k>=W_{k}(x)\right| k>,
$$

where

$$
<k^{\prime} \mid k>=\delta_{k^{\prime}, k},
$$

then a general wave function may be written in the adiabatic basis

$$
\left|\psi>=\sum_{k} C_{k}\right| k>
$$

The renormalized measurement Lagrangian for the classical apparatus may be defined as a mapping from an adiabatic basis state to an effective apparatus Lagrangian corresponding to that state; i.e.

$$
\mid k>\rightarrow \mathcal{L}_{k}(x, v)=L_{A}(x, v)-W_{k}(x),
$$

where $L_{A}(x, v)$ is defined in Eq.(2).

Quantum measurements are described as follows: (i) All data are classical. For a classical apparatus with coordinates $x$, the "data" are the "apparatus readings", i.e. classical paths $x(t)$. (ii) If the quantum object were in the state $\mid k>$, then the renormalized apparatus Lagrangian $\mathcal{L}_{k}(x, v)$ would control the classical path $x(t)$ via

$$
\mid k>\rightarrow\left\{\frac{d}{d t}\left(\frac{\partial \mathcal{L}_{k}}{\partial v^{k}}\right)=\left(\frac{\partial \mathcal{L}_{k}}{\partial x^{k}}\right)\right\} .
$$

(iii) The probability that the state $\mid k>$ is "observed" (i.e. that the apparatus yields a characteristic reading $x(t)$ from the Lagrangian $\mathcal{L}_{k}$ ) is given by

$$
P_{k}=\left|C_{k}\right|^{2}
$$

where the amplitude $C_{k}$ is defined in Eq.(20).
Thus, the quantum object Hamiltonian gives rise to a Lagrangian renormalization via Eqs.(18) and (21). The measurement process assigns to each adiabatic state $\mid k>$ of the measurement basis an apparatus Lagrangian $\mathcal{L}_{k}$ yielding a characteristic reading $x(t)$ from state $\mid k>$ with quantum probability $P_{k}=\left|C_{k}\right|^{2}$. This prescription is in agreement with the Bohr notion of how a quantum object yields a classical apparatus reading. This forms the basis of "quantum measurements" in which all "data" are classical in accordance with the so-called "Copenhagen School". A simple example of the above approach to measurements is the Stern-Gerlach experiment. Let us consider this in more detail.

\section{THE STERN-GERLACH EXPERIMENT}

As an example of the above formalism let us consider the Stern-Gerlach beam splitting experiment wherein a beam of spin one-half neutral atoms with a gyromagnetic ratio $\gamma$ passes through a spatial region with an inhomogeneous field $\mathbf{B}(\mathbf{r})$. The two by two spin Hamiltonian matrix corresponding to Eq.(5) reads (for this example) as

$$
H(\mathbf{r})=-\hbar \gamma \mathbf{S} \cdot \mathbf{B}(\mathbf{r}),
$$

while the eigenstates corresponding in Eq.(18) are determined by

$$
H(\mathbf{r})\left| \pm>=W_{ \pm}(\mathbf{r})\right| \pm>
$$

where

$$
W_{ \pm}(\mathbf{r})=\mp\left(\frac{\hbar \gamma|\mathbf{B}(\mathbf{r})|}{2}\right) .
$$

Thus, if the incoming beam is described as in Eq.(20) by the quantum state

$$
\left|\psi>=C_{+}\right|+>+C_{-} \mid->
$$

then with probability $P_{+}=\left|C_{+}\right|^{2}$ an atom in the beam follows the classical path specified by the classical equation of motion

$$
1+>\rightarrow\left\{M \frac{d^{2} \mathbf{r}}{d t^{2}}=\mathbf{F}_{+}(\mathbf{r})=-\nabla W_{+}(\mathbf{r})\right\},
$$

and with probability $P_{-}=\left|C_{-}\right|^{2}$ an atom in the beam follows the classical path specified by the classical equation of motion

$$
\mid->\rightarrow\left\{M \frac{d^{2} \mathbf{r}}{d t^{2}}=\mathbf{F}_{-}(\mathbf{r})=-\nabla W_{-}(\mathbf{r})\right\},
$$

The Stern-Gerach experiment represents the success of the Bohr theoretical interpretation of quantum mechanics at its very best. The quantum object is simply the spin $\mathbf{S}$ of the atom, which yields two possible states $\mid \pm>$. The classical apparatus reading is the path $\mathbf{r}(t)$ of the 
atom which obeys one of two possible classical \{Eqs.(28) or (29)\} of motion. All of this may be described by the adiabatic Lagrangians, as in Eq.(21)

$$
\mid \pm>\rightarrow \mathcal{L}_{ \pm}(\mathbf{r}, \mathbf{v})=\frac{1}{2} M \mathbf{v}^{2}-W_{ \pm}(\mathbf{r})
$$

where $W_{ \pm}(\mathbf{r})$ is defined in Eq.(26). Thus, the triumphant Bohr could predict that for a beam described by the quantum state in Eq.(27), with probability $\left|C_{ \pm}\right|^{2}$ one would find an atom of the beam moving along a classical path with a reading controlled by the Lagrangian $\mathcal{L}_{ \pm}(\mathbf{r}, \mathbf{v})$. The picture post card [5] with the note of congratulations from Gerlach to Bohr was at the start of an era of complete faith (if not blind then perhaps just near sighted) in quantum mechanics. The Copenhagan interpretation of quantum mechanics was perfect!

\section{WORK AND HEAT}

In the Lagrangian description of Sec.4, the effective Lagrangians were computed from the adiabatic energy levels $\left\{W_{k}(x)\right\}$. When the external coordinates are changed, the resulting induced changes in the adiabatic energy levels describe the thermodynamic notion of work done by the classical environment on the quantum object(or the reverse).

Another method of changing the energy of the quantum object, is by causing a transition from one adiabatic energy level to another adiabatic energy level. In thermodynamic terms such diabatic transitions describe the heat flow from the classical environment to the quantum object, (or the reverse).

Let us consider in more detail the decomposition of energy changes into "work" and "heat". The mean energy of quantum objects as members of a statistical ensemble may be written as

$$
\bar{E}=\operatorname{Tr}(\rho W),
$$

where $\rho$ is the density matrix of the ensemble which obeys

$$
i \hbar\left(\frac{\partial \rho}{\partial t}\right)=[\mathcal{H}, \rho],
$$

where $\mathcal{H}$ is defined in Eq.(13). From Eq.(31)

$$
\frac{d \bar{E}}{d t}=\operatorname{Tr}\left(\frac{\partial \rho}{\partial t} W\right)+\operatorname{Tr}\left(\rho \frac{\partial W}{\partial t}\right)
$$

which implies

$$
\frac{d \bar{E}}{d t}=-\frac{i}{\hbar} \operatorname{Tr}([\mathcal{H}, \rho] W)+\operatorname{Tr}\left(\rho \frac{\partial W}{\partial x^{k}}\right) v^{k},
$$

where Eq.(3) has been invoked. Note that the first term on the right hand side of Eq.(33) may be simplified according to

$$
\operatorname{Tr}([\mathcal{H}, \rho] W)=\operatorname{Tr}\left(\rho\left[W, v^{k} P_{k}\right]\right)
$$

where Eq.(13) has been invoked. Furthermore, from Eqs.(13), (16), (17), (34) and (35) it follows that

$$
\frac{d \bar{E}}{d t}=-\left(\bar{f}_{k}+\bar{F}_{k}\right) \frac{d x^{k}}{d t} .
$$

Eq.(36) is the central result of this section. The mean energy changes in the quantum object (as a member of a statistical ensemble) can be decomposed into heat and work; i.e.

$$
d \bar{E}=d^{\prime} Q+d^{\prime} W,
$$

where the work done on the quantum object by the classical environment is determined by

$$
d^{\prime} W=-\bar{F}_{k} d x^{k}
$$

and where the heat is determined by

$$
d^{\prime} Q=-\bar{f}_{k} d x^{k} .
$$

We employ the "inexact differential" $d^{\prime}$ notation for the heat $d^{\prime} Q$ (energy changes from quantum transitions between adiabatic energy levels) and the work $d^{\prime} W$ (energy changes from deformations in the given adiabatic energy levels). This decomposition of the mean energy change is exactly (i.e. rigorously) true, and constitutes a general derivation of the first law of thermodynamics.

\section{STATISTICAL THERMODYNAMICS}

The decomposition of energy changes into work and heat in Eqs.(37), (38) and (39) did not require that the quantum object be large or complex. An ensemble of two level atoms as used in the Stern-Gerlach atomic beam experiment is sufficient for the notion of introducing diabatic and adiabatic forces.

For the purpose of describing statistical thermodynamics, as employed for systems with a macroscopic number of particles, one normally introduces one (canonical or microcanonical) of the Gibbs probability distributions. For a macroscopic system, described by an infinite matrix $W(x)$, one may define $\Omega(E, x)$ as the number of quantum states with energy equal or less than $E$; i.e. with the step function

$$
\vartheta(E)= \begin{cases}1, & \text { if } E \geq 0 \\ 0, & \text { otherwise }\end{cases}
$$

we have

$$
\Omega(E, x)=\operatorname{Tr} \vartheta(E-W(x)) .
$$

The microcanonical entropy of the quantum system is then

$$
S(E, x)=k_{B} \ln \Omega(E, x) .
$$

The microcanonical temperature $T$, 


$$
\frac{1}{T}=\left(\frac{\partial S(E, x)}{\partial E}\right),
$$

is related to the microcanonical density of states,

$$
G(E, x)=\operatorname{Tr} \delta(E-W(x)),
$$

via

$$
\Omega(E, x)=k_{B} T(E, x) G(E, x) .
$$

In the thermodynamic limit of large quantum objects, the mean adiabatic force on the apparatus is given in the microcanonical ensemble by

$$
\bar{F}_{k}=-\left(\frac{\operatorname{Tr}\left(\delta(E-W)\left(\partial W / \partial x^{k}\right)\right)}{\operatorname{Tr} \delta(E-W)}\right),
$$

where Eqs.(16) has been invoked. From Eqs.(42)-(46), it follows that

$$
\bar{F}_{k}=T\left(\frac{\partial S}{\partial x^{k}}\right)_{E}=\left(\frac{\partial E}{\partial S}\right)_{x}\left(\frac{\partial S}{\partial x^{k}}\right)_{E},
$$

or equivalently

$$
\bar{F}_{k}(x, S)=-\left(\frac{\partial E(x, S)}{\partial x^{k}}\right) .
$$

For quasi-static changes in a macroscopic thermodynamic system we have in the microcanonical ensemble both the first and second law of thermodynamics in the form

$$
d E=T d S-\bar{F}_{k} d x^{k} .
$$

Note, from Eqs.(37), (38), (39), and (49), that the inexact heat differential from the diabatic forces has the temperature as an integrating factor

$$
d S=\left(\frac{d^{\prime} Q}{T}\right)=-\left(\frac{\bar{f}_{k} d x^{k}}{T}\right),
$$

as required by the second law implied existence of an entropy function. If one makes the Gibbs microcanonical assumption for quasi-static (sufficiently slow) changes, then one achieves the thermodynamic laws in a consistent manner.

However we have not thus far justified the Gibbs microcanonical assumption by anything deeper than the mere observation that this assumption is reasonable. It is here that we fall short of a strict mathematical derivation of the thermodynamic second law.

Our instinct is that one must consider a quantum object with an infinite number of degrees of freedom, e.g. as conventionally done in quantum field theory. For the field theory case, the states corresponding to different values of $x$ are not unitarily equivalent. Thus, there is no unitary transformation of the statistical state of the quantum object [6] when one changes the $x$ parameters from $x_{\text {inital }}$ to $x_{\text {final }} \neq x_{\text {inital }}$. While the usual unitary time development involves only the Hamiltonian, the necessarily diabatic time development between unitarily inequivalent Hilbert spaces involves also the entropy, or equivalently the free energy.

\section{FRICTION IN A MACROSCOPIC APPARATUS}

Let us now return to the apparatus viewed as having contact with a macroscopic thermodynamic system. In Eq.(2) we take the apparatus potential $V(x)$ to be determined by the apparatus adiabatic energy function $E_{A}(x, S)$; i.e.

$$
L_{A}(x, v)=\frac{1}{2} \mu_{j k}(x) v^{j} v^{k}-E_{A}(x, S),
$$

and we may further include the diabatic apparatus friction forces $\left\{\bar{f}_{A k}\right\}$ which heat the macroscopic degrees of freedom by

$$
\frac{d}{d t}\left(\frac{\partial L_{A}}{\partial v^{k}}\right)-\left(\frac{\partial L_{A}}{\partial x^{k}}\right)=\bar{f}_{A k} .
$$

For small velocities it is often sufficient to take the apparatus friction forces to be linear functions of the velocities,

$$
\bar{f}_{A k}=\Gamma_{k j}(x) v^{j} .
$$

Standard linear response theory gives expressions for the friction coefficients

$$
\Gamma_{k j}(x)=\frac{i}{\hbar} \int_{0}^{\infty}\left\langle\left[f_{k A}(x, t), P_{A j}(x)\right]\right\rangle d t,
$$

where Eqs.(13) and (17) has been invoked as well as the Heisenberg picture

$$
f_{A k}(x, t)=e^{i W_{A}(x) t / \hbar} f_{A k}(x) e^{-i W_{A}(x) t / \hbar} .
$$

If one employs the canonical density matrix,

$$
\rho_{A}=\frac{1}{Z_{A}} e^{-\beta W_{A}(x) / \hbar}, \beta=\left(\frac{\hbar}{k_{B} T}\right),
$$

which is equivalent to the microcanonical ensemble (for sufficiently large thermal systems), then the expression for the friction coefficients reduce to the Nyquist-Kubo formula [7],

$$
\begin{gathered}
\Gamma_{k j}(x)= \\
\frac{1}{\hbar} \int_{0}^{\beta} d \lambda \int_{0}^{\infty} d t\left\langle f_{j A}(x,-i \lambda) f_{k A}(x, t)\right\rangle,
\end{gathered}
$$

wherein the friction coefficient is related to the quantum fluctuations of the diabatic forces.

Thus in any apparatus one does expect some friction forces as in Eq.(52). The total apparatus heating rate

$$
\left(\frac{d^{\prime} Q_{A}}{d t}\right)=f_{A k} v^{k}
$$

in the linear regimes of Eq.(53) is described by the Rayliegh dissipation which is quadratic in the velocities 


$$
\left(\frac{d^{\prime} Q_{A}}{d t}\right)=\Gamma_{k j}(x) v^{k} v^{j} .
$$

For the case of the Stern-Gerlach experiment, the apparatus friction forces on the atoms with spin are quite small. The dissipation in this experiment amounts to the small friction forces induced by Ohms law eddy currents inside the deflecting magnets in the beam splitter.

\section{LOW VELOCITY PROJECTIVE MEASUREMENTS ARE FRICTIONLESS}

Within the above discussion of quantum measurements in the adiabatic representation of Eq.(10), the statistical state of a quantum object is described by the matrix

$$
\rho=\left(\begin{array}{cccc}
\rho_{11} & \rho_{12} & \ldots & \rho_{1 m} \\
\rho_{21} & \rho_{22} & \ldots & \rho_{2 m} \\
\ldots & \ldots & \ldots & \ldots \\
\rho_{m 1} & \rho_{m 2} & \ldots & \rho_{m m}
\end{array}\right) .
$$

The unitary time development of the statistical state obeys the equation of motion

$$
i \hbar\left(\frac{\partial \rho}{\partial t}\right)=[\mathcal{H}, \rho]
$$

where $\mathcal{H}$ is given in Eq.(13). However, in the fairly early days of quantum mechanics, von Neumann introduced, over and above the standard Bohr Copenhagen interpretation, the notion of a projective measurement [8]. During a projective measurement, the density matrix is thought to obey a non-unitary time development governed by

$$
\rho \rightarrow \rho_{P}=\sum_{k} P_{k} \rho P_{k},
$$

for a set of projection operators $P_{k}=P_{k}^{\dagger}=P_{k}^{2}$ which is complete $\sum_{k} P_{k}=1$. For the problem at hand $P_{k}=$ $|k><k|$, so that the projected matrix

$$
\rho \rightarrow \rho_{P}=\left(\begin{array}{cccc}
\rho_{11} & 0 & \ldots & 0 \\
0 & \rho_{22} & \ldots & 0 \\
\ldots & \ldots & \ldots & \ldots \\
0 & 0 & \ldots & \rho_{m m}
\end{array}\right)
$$

has the above diagonal form.

Note, from Eqs.(10), (17) and (63), it follows that the mean diabatic (frictional heating) force on the quantum object obeys

$$
\bar{f}_{P k}=\operatorname{Tr}\left(\rho_{P} f_{k}\right)=0 .
$$

The frictional force is zero, when computed from the von Neumann projected density matrix. No diabatic heat is generated in a low velocity projective measurement. This strongly suggests that projective measurements (in general) violate energy conservation, which is indeed true.
Suppose that the projection operator does not commute with the total energy operator of the apparatus plus the quantum object. Further suppose that before the measurement the system plus apparatus have a sharply defined energy. After the measurement the energy value will no longer be sharp since the projection operator does not commute with the total energy. Thus, with finite probability, the total energy is not conserved. We consider this violation to be a significant flaw in the projective measurement picture. Let us reconsider the situation without imposing the von Neumann projection postulate.

In the Heisenberg (unitary time development) picture, the diabatic friction force operator obeys

$$
\left(\frac{d f_{k}}{d t}\right)=\left(\frac{\partial f_{k}}{\partial t}\right)+\frac{i}{\hbar}\left[\mathcal{H}, f_{k}\right] .
$$

With adiabatic Bohr transition frequencies

$$
\hbar \omega_{i j}(x)=W_{i}(x)-W_{j}(x),
$$

and for a slowly moving apparatus (small $v^{k}$ ), the diabatic friction force operator has only quickly oscillating off diagonal matrix elements

$$
<j\left|f_{k}(t)\right| i>\sim e^{-i \int \omega_{i j}(x(t)) d t}<j\left|f_{k}\right| i>
$$

whose time average is virtually zero. Again, in a time averaged sense, the mean diabatic friction force is negligible, $\bar{f}_{k} \approx 0$. For the case of a Stern-Gerlach experiment, the time averaged null behavior of the rapidly varying diabatic friction force has been discussed elsewhere 9 .

Thus, in the general case, the mean energy changes of an ensemble of quantum objects decomposes into a "heat" and "work" term

$$
d \bar{E}=d^{\prime} Q+d^{\prime} W
$$

with the inequality

$$
\left|d^{\prime} Q\right|<<\left|d^{\prime} W\right|, \text { projective measurement. }
$$

We stress that the diabatic friction forces can be small (on the average) if the apparatus velocities are also small. However, even if the diabatic forces during a projective measurement on the average are small, they are often still present as "noise". Via the fluctuation-dissipation Eq.(57), such noise does give rise to friction at least for higher velocities if the apparatus is (at least in part) macroscopic. In the limit of an infinite number of degrees of freedom, one is not allowed to totally ignore heating, since (as discussed in Sec.7) the diabatic forces are invoked as part of the dynamics of passing between two unitarily inequivalent spaces.

\section{INFORMATION AND ENTROPY}

In some treatments of statistical thermodynamics, one associates the physical entropy to the statistical "information" contained in $\rho$ [8] [10] [11]; i.e. 


$$
\mathcal{S}=-k_{B} \operatorname{Tr}(\rho \ln \rho) .
$$

The difficulty with this approach is that the information entropy $\mathcal{S}$ is not always simply related to thermodynamic heat $d^{\prime} Q=-\bar{f}_{k} d x^{k}$. For example, von Neumann has proved [8] for the projective measurement in Eq.(62), that

$$
\mathcal{S}_{P}=-k_{B} \operatorname{Tr}\left(\rho_{P} \ln \rho_{P}\right)
$$

and that the change in statistical entropy obeys

$$
\Delta \mathcal{S}=\mathcal{S}_{P}-\mathcal{S} \geq 0
$$

Thus the statistical entropy of a projective measurement is thought to increase, yet the frictional heating (i.e. thermodynamic entropy increase $\left.d^{\prime} Q / T\right)$ in a low velocity projective measurement is virtually zero.

The paradox may be resolved when it is realized that during the unitary time development of Eq.(61), it is in fact rigorously true that the statistical entropy of Eq.(70) does not increase at all; i.e.

$$
\frac{d \mathcal{S}}{d t}=-k_{B} \frac{d}{d t} \operatorname{Tr}(\rho \ln \rho)=0 .
$$

Thus it is only through the projection postulate itself, that the statistical entropy may be shown to increase.

Finally, non-unitary time development can enforce the approximate equality of the thermodynamic entropy in Eq.(42) and the statistical entropy Eq.(70) as in Sec.7. This is likely to happen in the limit of an infinite number of degrees of freedom. It is in such a limit that the information theory picture may be properly formulated, also with possible advantage in problems of quantum computation [12].

\section{CONCLUSIONS}

In quantum mechanics, the unitary time development of the statistical state $\rho$ is all that we know for sure to be true. Under some circumstances, this density matrix $\rho$ may be approximated by a projected density matrix $\rho_{P}$. The reason why this replacement may not lead to error, is that there exists very rapid phase oscillations which average out (e.g. for the diabatic forces) and which cannot later be resolved experimentally.

If such measurements are statistically accurately described by the projection postulate, via $\rho \rightarrow \rho_{P}$, then there is a quantity which we have called statistical entropy which increases, as was early proved by von Neumann. A difficulty with this definition of entropy, via information theory, is that the statistical entropy is not always simply related to the thermodynamic notion of heat $d^{\prime} Q=-\bar{f}_{k} d x^{k}$. For the case of the (macroscopically equivalent) Gibbs ensembles, the statistical entropy is closely related to heating $d^{\prime} Q=T d S$. The reasoning applied to the Gibbs ensembles is that statistical entropy is at maximum (subject to conservation law constraints). Then the statistical entropy does indeed obey $d^{\prime} Q=T d S$, but then there is little difference between the statistical entropy and the thermodynamic $S=k_{B} \ln \Omega$.

If the quantum object has an infinite number of degrees of freedom, it is reasonable to invoke a non-unitary time development between Hilbert spaces which are not unitarily equivalent. For this situation, it appears reasonable that the equivalence between the statistical entropy and the thermodynamic entropy may be established during irreversible dynamics.

Although we do not pretend that a complete proof of the physical picture has been provided, we feel that the discussion presented in this work accurately describes the problem of understanding the connection between quantum measurements and the second thermodynamic law of entropy increase.

This work has been partially supported by the INFN, INFM and MURST.

[1] A. Fine, The Shaky Game: Einstein, Realism and Quantum Theory, University of Chicago Press, Chicago (1986).

[2] L. D. Landau and E. M. Lifshitz, Quantum Mechanics: Non-Relativistic Theory, Pergamon Press, Oxford, (1977).

[3] L. D. Landau and E. M. Lifshitz, Statitsical Physics, Pergamon Press, Oxford, (1978).

[4] Von W. Gerlach and O. Stern, Z. Phys. 8, 110, (1921).

[5] J. S. Townsend, A modern Approach to Quantum Mechanics, McGraw Hill Inc., New York, (1992).

[6] M. Blasone, Y. N. Srivastava, G. Vitiello and A. Widom, Annals of Phys. 267, 61, (1998).

[7] M. Toda, R. Kubo and N. Saito, Statistical Physics 2, Springer Verlag, New York (1991).

[8] J. von Neumann, Mathematical Foundations of Quantum Mechanics, Princeton University Press, Princeton (1955).

[9] M. Hannout, S. Hoyt, A. Kryowonos and A. Widom, Am. J. Phys. 66, 377, (1998).

[10] E. T. Jaynes, Phys. Rev. 106, 620 (1957); Phys. Rev. 108, 171 (1957).

[11] H. S. Leff and A. F. Rex (Eds.), Maxwell's Demon: Entropy, Information, Computing, Princeton University Press, Princeton (1988).

[12] C. P. Williams and S. H . Clearwater Explorations in Quantum Computing, Springer-Verlag, New York (1998). 